\title{
The influence of anesthesia on intraoperative neurophysiological monitoring during spinal surgeries
}

\author{
Benuska J, Plisova M, Zabka M, Horvath J, Tisovsky P, Novorolsky K \\ Department of Orthopedic and Trauma Surgery, Comenius University Hospital, Bratislava, Slovakia. \\ drbenuska@gmail.com
}

\begin{abstract}
BACKGROUND: Intraoperative neuromonitoring using tc-MEPs satisfactorily detects motor tract integrity changes during spinal surgery. However, tc-MEP is affected by anesthesia and other factors, in which the stimulation threshold increases because the waveform amplitude decreases over time with the accumulation and boluses of anesthetics.

METHODS: We conducted a retrospective study of 139 patients. The average age was 30 years. Tc-MEPs were recorded bilaterally from the tibialis anterior muscle and the abductor hallucis muscle. Statistical tests were used to investigate the changes to evaluate anesthetic effects.

RESULTS: There were no significant differences in tc-MEP amplitude change (\%) between the groups of propofol $(13 \%)$, remifentanil $(22 \%)$ and sufentanil $(26 \%, p<0.01)$. Significant differences were found between the groups of propofol, remifentanil, and sufentanil (20\%) and bolus sufentanil ( $-30 \%)$, and bolus ketamine $(730 \%, p<0.008)$. Major differences were observed between bolus sufentanil $(-30 \%)$ and bolus ketamine $(730 \%, p<0.001)$. When comparing tc-MEPs with no amplitude, no significant difference was found between the groups of propofol $(26 \%)$, remifentanil $(24 \%)$, and sufentanil $(28 \%, p<0.007)$. Substantial difference was found between the groups of propofol, remifentanil, and sufentanil (mean $26 \%$ ) and the group where ketamine boluses were administered. We didn't observe any loss of amplitude $(0 \%, p<0.0002)$. CONCLUSION: IONM may be useless in patients where boluses of sufentanil are administered and also with Medical Research Council grades 3 and below. Consider applying IONM in patients with severe spinal deformity along with a higher age of over 50 and neurological deficit. Increasing stimulus intensity or facilitation techniques may be considered to improve the usefulness of tc-MEP. Our concept of findings supports the neurophysiological monitoring findings in other studies (Tab. 10, Ref. 45). Text in PDF www.elis.sk. KEY WORDS: neuromonitoring, MEP, motor evoked potential, spinal deformity, anesthesia, propofol, sufentanil, ketamine, amplitude.
\end{abstract}

\section{Introduction}

Spinal surgery with intraoperative neurophysiological monitoring (IONM) is advancing and becoming prevalent (1-3). Transcranial motor evoked potentials (tc-MEPs) are universally recorded during intraoperative monitoring to ensure the integrity of the descending spinal cord, but indications in lower risk decompressive surgeries are more controversial (4). The integrity of the spinal cord is the main assessment of electrophysiologic monitoring together with the entire team participating in the operation of the spine to recognize and avoid injury to the spinal cord (5). Despite all this, tc-MEPs have excellent sensitivity for detecting changes in motor tract integrity during spinal surgery (6-8). In general, patients with scoliosis are neurologically intact, compared to those who

Department of Orthopedic and Trauma Surgery, Comenius University Hospital, Bratislava, Slovakia

Address for correspondence: J. Benuska, MD, Department of Orthopedic and Trauma Surgery, University Hospital Bratislava, Ruzinov, Ruzinovska 6, SK-826 06 Bratislava, Slovakia.

Phone: +421.2.48234724, Fax: +421.2.48234313 are undergoing surgery for intramedullary or extramedullary spinal cord disorders. MEPs are susceptible to the effects of perioperative environmental factors. Amplitude in MEPs can decrease even in the absence of any nerve damage (false positive), caused by intraoperative hypotension, hypothermia or electrode failure $(9,10)$. Volatile anesthetic agents suppress the excitability of the motor cortex, resulting in diminished amplitudes $(11,12)$. To decrease inhalational anesthetic effects, it is recommended to apply total intravenous anesthesia (TIVA) instead of volatile anesthetics $(11,13)$. The intraoperative application of muscle relaxants solely following intubation has been suggested as the preferred anesthetic technique for surgeries with MEPs (14). It has been reported that anesthetic agents such as propofol suppress MEPs, while intravenous ketamine (ket) does not suppress MEPs and may even facilitate them (15-19). In a dose dependent fashion, in addition most anesthetics, other than remifentanil and ketamine, can cause false positive results by inhibiting MEPs (20). Optimal conditions and an adequate depth of anesthesia ensure TIVA using a propofol infusion along with stable blood pressure, core temperature and avoidance of excessive blood loss (21-23). Other published studies showed fading MEP with prolonged exposure to anesthesia 
described as "anesthetic fade" (5). In his study, MacDonald et al (24) found abrupt lower limb MEP loss during prolonged scoliosis surgery restored after instrumentation release without neurologic deficit and suggested increasing MEP stimulation parameters to offset this effect. There is limited information on whether a balanced technique of anesthesia improves increased IONM changes. There is also a paucity of information on the intraoperative anesthetic and surgical interventions performed on notification of an IONM change (25). Holdefer et al found no evidence of a reducing trend of amplitude over time (26). This has been confirmed by another study which also found no significant change of MEP amplitude over $120 \mathrm{~min}$. during surgery of propofol anesthesia (27). Surgeons should understand the rationale and clinical basis for IONM to interpret the monitoring changes and to utilize them for a better surgical outcome.

The purpose of this study was to compare several different groups of patients, and how the type of spinal deformity and contingency of neurological status versus different types of anesthesia ultimately affect the tc-MEP waveforms.

\section{Materials and methods}

Approval of the study was obtained by the institution's ethics committee of University Hospital Bratislava, Slovakia (EK/62/2019). No patient consent was deemed necessary. All records and personal data of patients were anonymized.

\section{Study design}

We conducted a retrospective study of 139 patients between January 2007 and January 2018. All of these patients underwent corrective spinal surgery in our department together with IONM requiring tc-MEP indication as determined by the operating surgeon. Patients ranged in age from 12 to 79 years (standard deviation, mean 18). Regarding sex, there were 22 males and 117 females in the study. The inclusion criteria were all the patients that had a tc-MEP performed during spinal surgery. There were no exclusion criteria (Tab. 1).

\section{Data source collection}

The electronic and paper patient medical data were reviewed for all 139 patients. The data collected included general patient information (age, height, weight, sex, date of study) (Tab. 2), anesthesiology data (type, hemodynamics, physiological values), and surgical as well as neuromonitoring information (type of spinal deformity, procedure, monitoring techniques, events in tc-MEP change for each electrode separately, variables).

The types of spinal deformities were divided into six groups and a subgroup of neurological deficit alone (Tab. 3). The surgi-

\section{Tab. 1. Patient selection, inclusion and exclusion criteria.}

\begin{tabular}{l}
\hline Inclusion criteria \\
Patients who underwent a surgical procedure for spinal surgery with \\
IONM of tc-MEP \\
\hline Exclusion criteria \\
Depends on each group, subgroup \\
\hline
\end{tabular}

Tab. 2. Patient characteristics according to groups of spinal deformities and neurological deficit subgroup.

\begin{tabular}{lcccccc}
\hline $\begin{array}{l}\text { Groups of } \\
\text { patients }\end{array}$ & $\begin{array}{c}\text { number } \\
(\mathrm{n})\end{array}$ & $\begin{array}{c}\text { age } \\
(\text { years })\end{array}$ & $\begin{array}{c}\text { weight } \\
(\mathrm{kg})\end{array}$ & $\begin{array}{c}\text { height } \\
(\mathrm{cm})\end{array}$ & $\begin{array}{c}\text { sex } \\
(\mathrm{M} / \mathrm{F})\end{array}$ & $\begin{array}{c}\text { hgb } \\
(\mathrm{g} / \mathrm{l})\end{array}$ \\
\hline Total & 139 & 27 & 59 & 165 & $22 / 117$ & $44.3 \searrow$ \\
AIS2 & 64 & 17 & 54 & 166 & $4 / 60$ & $45.4 \searrow$ \\
AIS3 & 27 & 19 & 54 & 162 & $4 / 23$ & $49.6 \searrow$ \\
AIS4 & 3 & 16 & 51 & 163 & $0 / 3$ & $42.7 \searrow$ \\
N & 8 & 19 & 50 & 162 & $2 / 6$ & $52.0 \searrow$ \\
D & 16 & 55 & 66 & 164 & $2 / 14$ & $37.3 \searrow$ \\
I & 21 & 51 & 77 & 170 & $10 / 11$ & $38.8 \searrow$ \\
Neurological & 43 & 48 & 62 & 162 & $10 / 33$ & $42.2 \searrow$ \\
deficit (ND) & & & & & &
\end{tabular}

Values are presented as a number. $\searrow$ indicates a decrease of hgb during the entire anesthesia time.

Tab. 3. Groups of spinal deformities and neurological alteration subgroup.

Total - complete sample of all patients in all groups

AIS2-adolescent idiopathic scoliosis grade II, (Cobb angle $\left.=30^{\circ}-60^{\circ}\right)$

AIS3 -adolescent idiopathic scoliosis grade III, (Cobb angle $\left.=60^{\circ}-90^{\circ}\right)$

AIS4-adolescent idiopathic scoliosis grade IV, $\left(\mathrm{Cobb}\right.$ angle $\left.>90^{\circ}\right)$

$\mathrm{D}$-degenerative scoliosis all grades,

$\mathrm{N}-$ neuromuscular scoliosis all grades,

I-other spinal deformities (metastases, spinal stenosis, herniated discs, other) $\mathrm{ND}$ - neurological deficit (separate subgroup of patients with ND before or after spinal surgery)

cal procedure in the AIS2-4, neuromuscular and degenerative scoliosis groups was usually performed with dorsal transpedicular stabilization with correction of scoliosis. Severe scoliotic curves were treated with combined surgical procedures that consisted of osteotomies and lateral approaches. Other spinal deformities (I), usually degenerative, were treated with dorsal transpedicular stabilization along with laminectomy and spinal decompression. Complications and/or post-operative neurological outcomes were also collected in the neurological deficit (ND) subgroup. We used Medical Research Council (MRC) grading. Modification of the MRC grading was made as we added a sensory deficit (MRC 5 normal strength with sensory deficit) and radicular pain (MRC 6 normal strength with radicular pain).

\section{Anesthetic management}

In all patients, anesthesia was maintained with total intravenous anesthesia (TIVA) during the period of MEPs. A muscle relaxant, rocuronium bromide (Esmeron 30-50 mg), was administered before intubation. Perioperatively, after induction and intubation, all muscle relaxants were discontinued during surgery. An arterial line was placed to monitor blood pressure. Other standard monitoring, such as electrocardiogram and oxygen saturation was managed by the anesthesiologist.

The patients were classified into five groups: propofol; propofol with remifentanil; propofol with sufentanil; propofol with bolus sufentanil; propofol with ketamine bolus (Tab. 4). Propofol, sufentanil and remifentanil were administered as shown (Tab. 5). Sufentanil boluses were administered in doses of $3 \mathrm{ml}$ intravenously $(5 \mu \mathrm{g} / \mathrm{ml})$. Ketamine boluses were administered in doses of $30 \mathrm{mg}$ intravenously $(50 \mathrm{mg} / \mathrm{ml})$. 


\section{4-801}

Tab. 4. Patient characteristics in correlation with anesthetics.

\begin{tabular}{|c|c|c|c|c|c|c|}
\hline Anesthetics & $\begin{array}{l}\text { Number of patients } \\
\text { (n) }\end{array}$ & $\begin{array}{c}\text { Age } \\
\text { mean (years) }\end{array}$ & $\begin{array}{c}\text { Weight } \\
\text { mean }(\mathrm{kg})\end{array}$ & $\begin{array}{c}\text { Height } \\
\text { mean }(\mathrm{cm})\end{array}$ & $\begin{array}{c}\text { Sex } \\
\text { No. of patients }(M / F)\end{array}$ & $\begin{array}{c}\mathrm{Hgb} \\
\text { mean }(\mathrm{g} / \mathrm{l})\end{array}$ \\
\hline Total/Propofol & $139(100)$ & 30 & 59 & 165 & $22(16) / 117(84)$ & 44\(34) \\
\hline Remifentanil & $75(54)$ & 29 & 60 & 166 & $10 /(13) / 65(87)$ & $45 \searrow(34)$ \\
\hline Sufentanil & $64(46)$ & 25 & 57 & 166 & $12(19) / 52(81)$ & 44】(34) \\
\hline Bolus sufentanil & $26(19)$ & 25 & 57 & 166 & $3(12) / 23(88)$ & $46 \searrow(34)$ \\
\hline Bolus ketamine & $28(20)$ & 18 & 54 & 164 & $1(4) / 27(96)$ & $45 \searrow(34)$ \\
\hline
\end{tabular}

Values are presented as a number (\%). $\nearrow$ indicates an increase, $\searrow$ indicates a decrease.

Tab. 5. Mean administered doses of anesthetics according to groups of spinal deformities.

\begin{tabular}{lccc}
\hline Groups & propofol & sufentanil & remifentanil \\
\hline Total & $6.07 \mathrm{mg} / \mathrm{kg} / \mathrm{h}$ & $0.44 \mu \mathrm{g} / \mathrm{kg} / \mathrm{h}$ & $0.21 \mu \mathrm{g} / \mathrm{kg} / \mathrm{min}$ \\
AIS2 & $6.37 \mathrm{mg} / \mathrm{kg} / \mathrm{h}$ & $0.42 \mu \mathrm{g} / \mathrm{kg} / \mathrm{h}$ & $0.25 \mu \mathrm{g} / \mathrm{kg} / \mathrm{min}$ \\
AIS3 & $6.31 \mathrm{mg} / \mathrm{kg} / \mathrm{h}$ & $0.43 \mu \mathrm{g} / \mathrm{kg} / \mathrm{h}$ & $0.22 \mu \mathrm{g} / \mathrm{kg} / \mathrm{min}$ \\
AIS4 & $5.53 \mathrm{mg} / \mathrm{kg} / \mathrm{h}$ & $0.39 \mu \mathrm{g} / \mathrm{kg} / \mathrm{h}$ & $0.25 \mu \mathrm{g} / \mathrm{kg} / \mathrm{min}$ \\
N & $5.62 \mathrm{mg} / \mathrm{kg} / \mathrm{h}$ & $0.63 \mu \mathrm{g} / \mathrm{kg} / \mathrm{h}$ & $0.27 \mu \mathrm{g} / \mathrm{kg} / \mathrm{min}$ \\
D & $5.02 \mathrm{mg} / \mathrm{kg} / \mathrm{h}$ & $0.32 \mu \mathrm{g} / \mathrm{kg} / \mathrm{h}$ & $0.13 \mu \mathrm{g} / \mathrm{kg} / \mathrm{min}$ \\
I & $6.64 \mathrm{mg} / \mathrm{kg} / \mathrm{h}$ & $0.52 \mu \mathrm{g} / \mathrm{kg} / \mathrm{h}$ & $0.14 \mu \mathrm{g} / \mathrm{kg} / \mathrm{min}$ \\
\hline
\end{tabular}

Values are presented as a number.

\section{Electrophysiology data analysis}

Intraoperative neuromonitoring was performed according to international guidelines. Baseline MEPs were obtained after positioning the patient. The IONM was performed with a Medtronic Dantec KeyPoint EMG/EP 4 channel amplifier and 2 channel stimulator. Tc-MEPs were generated by a multipulse generator and delivered to two sensory needle electrodes $(28 \mathrm{G})$ placed over the $\mathrm{C} 3$ and $\mathrm{C} 4$ motor cortical regions according to the international $10-20$ system. Electrical stimulation was set to $100 \mathrm{~mA}, 2.5 \mathrm{~ms}$ and an amplitude of $0.1 \mathrm{~Hz}$ in all patients. Electromyographic responses were recorded from pre-gelled surface-patched electrodes placed bilaterally on the tibialis anterior muscle and the abductor hallucis muscle. Waveforms measured in $\mu \mathrm{V}$ were recorded and evaluated on Medtronic Dantec KeyPoint.net software. Input impedance of stimulating and recording electrodes was usually maintained below $5 \mathrm{k} \Omega$. We took actions to recover deteriorated MEPs as shown by other authors (28-33). We reviewed the MEP records from the lower extremities and investigated the change of amplitude from the baseline to the end of IONM in all spinal deformity groups
(Tab. 6). Also, we aimed the amplitude recording in groups with boluses of sufentanil and ketamine after an interval of 30 minutes.

\section{Data analysis}

The data were analyzed to allocate the incidence of neuromonitoring intraoperative changes in correlation to anesthesia. We considered all amplitude changes that occurred. We compared the differences in the groups. The main point of this study was to compare the remifentanil and sufentanil groups and the groups with bolus of sufentanil and bolus of ketamine. Our suspicion of statistically significant results was considered with the Fisher ftest and students t-test, we also used the ANOVA test for larger groups (34). Values of probability p-value lower than 0.05 were considered statistically significant. All analyses were performed using Microsoft Excel 2007 with the Analysis ToolPak.

\section{Results}

In our comprehensive study, we retrospectively observed and analyzed several groups with different anesthetic regimens over 10 years. We compared magnitudes of tc-MEP waveforms and anesthetic regimens (Tabs 7-10). We were interested in identifying specific groups with significant associations as follows.

\section{Anesthesia regimen in association with tc-MEP, outcome}

There were no significant differences in tc-MEP amplitude change between the groups of propofol (13\% increase), remifentanil (22\% increase), and sufentanil ( $26 \%$ increase, $\mathrm{p}<0.01)$. Significant differences were found between the groups of propofol,

Tab. 6. Spinal deformity groups and neurological deficit subgroup in dependence on tc-MEPs.

\begin{tabular}{|c|c|c|c|c|c|c|c|c|}
\hline \multirow[b]{2}{*}{$\begin{array}{l}\text { Deformity } \\
\text { types }\end{array}$} & \multirow[b]{2}{*}{$\begin{array}{c}\text { Number } \\
\text { of patients (n) }\end{array}$} & \multirow[b]{2}{*}{$\begin{array}{l}\text { Age mean } \\
\text { (years) }\end{array}$} & \multicolumn{6}{|c|}{ tc-MEP waveform } \\
\hline & & & $\begin{array}{c}\text { mean base } \\
(\mu \mathrm{V})\end{array}$ & $\begin{array}{c}\text { mean end } \\
(\mu \mathrm{V})\end{array}$ & $\begin{array}{c}\max . \text { base/end } \\
(\mu \mathrm{V})\end{array}$ & $\begin{array}{c}\text { min. base/end } \\
(\mu \mathrm{V})\end{array}$ & $\begin{array}{l}\text { absolute change } \\
(\mu \mathrm{V})\end{array}$ & $\begin{array}{c}\mu \mathrm{V} \text { change } \\
\text { base-end line }\end{array}$ \\
\hline Total & $139(100)$ & 27 & 203 & 225 & $578 / 443$ & $55 / 9$ & $22 \pi$ & $13 \% \pi$ \\
\hline AIS2 & $64(46)$ & 17 & 218 & 301 & $928 / 1195$ & $5 / 4$ & $83 \pi$ & $41 \% \nearrow$ \\
\hline AIS3 & $27(19)$ & 19 & 186 & 165 & $793 / 596$ & $14 / 5$ & 21】 & $7 \% \searrow$ \\
\hline AIS4 & $3(2)$ & 16 & 218 & 0 & $218 / 0$ & $218 / 0$ & $218 \searrow$ & $75 \% \searrow$ \\
\hline $\mathrm{N}$ & $8(6)$ & 19 & 149 & 95 & $419 / 205$ & $25 / 29$ & $53 \searrow$ & $8 \% \searrow$ \\
\hline $\mathrm{D}$ & $16(12)$ & 55 & 122 & 107 & $490 / 418$ & $9 / 10$ & $15 \searrow$ & $6 \% \searrow$ \\
\hline I & $21(15)$ & 51 & 285 & 70 & $619 / 243$ & $58 / 7$ & 216】 & $73 \% \searrow$ \\
\hline ND & $43(31)$ & 48 & 156 & 101 & $424 / 418$ & $17 / 8$ & $55 \searrow$ & $35 \% \searrow$ \\
\hline
\end{tabular}

Values are presented as a number (\%). Baseline mean value and endline of tc-MEPs. Absolute change column indicates $\nearrow$ amplitude increase or $\searrow$ amplitude decrease of tc-MEPs from the beginning to the endline. In the ND subgroup we didn't consider tc-MEPs with no amplitude 
Tab. 7. Anesthesia regimen in association with/without tc-MEPs between spinal deformities.

\begin{tabular}{|c|c|c|c|c|c|c|c|c|}
\hline \multirow{2}{*}{ Anesthetics } & AIS2 & AIS3 & AIS4 & $\mathrm{N}$ & $\mathrm{D}$ & I & ND & Total \\
\hline & \multicolumn{8}{|c|}{ mean tc-MEP magnitude baseline/endline } \\
\hline \multicolumn{9}{|l|}{ Propofol } \\
\hline TA 1.sin. & $83 \nearrow(40)$ & $16 \searrow(11)$ & $0(0)$ & $44 \nearrow(78)$ & $37 \searrow(37)$ & $80 \searrow(37)$ & $25 \searrow(34)$ & $42 \nearrow(24)$ \\
\hline AH 1.sin. & $99 \lambda(65)$ & $72 \searrow(31)$ & $266 \searrow(100)$ & $102 \searrow(70)$ & $46 \lambda(57)$ & $240 \unlhd(76)$ & $27 \searrow(24)$ & $21>(12)$ \\
\hline TA 1.dx. & $87 \nearrow(37)$ & $34 \nearrow(28)$ & $488 \searrow(100)$ & $41 \nearrow(34)$ & $37 \searrow(24)$ & $212 \searrow(88)$ & $12 \searrow(22)$ & $38 \nearrow(19)$ \\
\hline AH 1.dx. & $62 \pi(22)$ & $28 \searrow(12)$ & $118 \searrow(100)$ & $196 \searrow(72)$ & $33 \searrow(21)$ & $330 \searrow(90)$ & $65 \searrow(17)$ & $11 \searrow(4)$ \\
\hline mean & $83 \nearrow(41)$ & $21 \searrow(7)$ & $218 \searrow(100)$ & $53 \searrow(8)$ & $15 \searrow(6)$ & $216 \searrow(73)$ & $32 \searrow(24)$ & $22 \nearrow(13)$ \\
\hline & \multicolumn{8}{|c|}{$\begin{array}{l}\text { without tc-MEP response (no amplitude presence) } \\
\text { baseline/endline }\end{array}$} \\
\hline & \multicolumn{8}{|c|}{ Number of patients ( $\%$ ) } \\
\hline TA 1.sin. & $2(3) / 9(14)$ & $6(22) / 8(30)$ & $3(100) / 3(100)$ & $1(13) / 2(25)$ & $6(38) / 6(38)$ & $13(62) / 12(57)$ & $20(47) / 22(51)$ & $32(23) / 48(35)$ \\
\hline AH 1.sin. & $3(5) / 8(13)$ & $4(15) / 4(15)$ & $2(67) / 2(67)$ & $1(13) / 1(13)$ & $5(31) / 5(31)$ & $13(62) / 12(57)$ & $19(44) / 20(47)$ & $29(21) / 39(28)$ \\
\hline TA 1.dx & $3(5) / 4(6)$ & $4(15) / 3(11)$ & $2(67) / 2(67)$ & $2(25) / 3(38)$ & $6(38) / 6(38)$ & $12(57) / 12(57)$ & $20(47) / 19(44)$ & $30(22) / 36(26)$ \\
\hline AH 1.dx. & $4(6) / 9(14)$ & $4(15) / 3(11)$ & $2(67) / 2(67)$ & $2(25) / 3(38)$ & $5(31) / 5(31)$ & $12(57) / 12(57)$ & $20(47) / 18(42)$ & $30(22) / 42(30)$ \\
\hline Total mean & $8 \%$ & $17 \%$ & $75 \%$ & $23 \%$ & $34 \%$ & $58 \%$ & $46 \%$ & $26 \%$ \\
\hline \multicolumn{9}{|l|}{ Tc-MEP depend. on time } \\
\hline$\mu \mathrm{V} /$ anesthesia time & $52 \pi / 287$ & $26 \searrow / 317$ & $218 \searrow / 370$ & $71 \searrow / 358$ & $25 \searrow / 264$ & $151 \searrow / 237$ & $45 \searrow / 279$ & $73 \searrow / 288$ \\
\hline$\mu \mathrm{V} /$ minute & $0.18 \nearrow$ & $0.08 \searrow$ & $0.59 \searrow$ & $0.20 \searrow$ & 0.10】 & $0.64 \searrow$ & $0.16 \searrow$ & $0.25 \searrow$ \\
\hline & \multicolumn{8}{|c|}{ mean tc-MEP magnitude baseline/endline } \\
\hline \multicolumn{9}{|l|}{ Remifentanil } \\
\hline TA 1.sin. & $27 \nearrow(15)$ & $21 \searrow(16)$ & $0 \searrow(0)$ & $4 \nearrow(8)$ & $65 \searrow(55)$ & $9 \nearrow(7)$ & $28 \searrow(15)$ & $5 \nearrow(3)$ \\
\hline AH 1.sin. & $50 \nearrow(36)$ & $81 \searrow(37)$ & $0 \searrow(0)$ & $90 \searrow(79)$ & $31 \searrow(33)$ & $218 \searrow(77)$ & $25 \searrow(14) \nearrow$ & $13 \searrow(8)$ \\
\hline TA 1.dx. & $82 \nearrow(41)$ & $59 \nearrow(59)$ & $0 \searrow(0)$ & $29 \nearrow(31)$ & $93 \searrow(58)$ & $349 \searrow(93)$ & $28 \searrow(15)$ & $24 \nearrow(14)$ \\
\hline AH 1.dx. & $3 \nearrow(1)$ & $25 \nearrow(13)$ & $0 \searrow(0)$ & $115 \searrow(55)$ & $58 \searrow(35)$ & $466 \searrow(93)$ & $100 \searrow(21)$ & $32 \searrow(12)$ \\
\hline mean & $41 \nearrow(23)$ & $4 \searrow(5) \nearrow$ & $0 \searrow(0)$ & $43 \searrow(24)$ & $62 \searrow(45)$ & $256 \searrow(64)$ & $45 \searrow(9)$ & $4 \searrow(22) \nearrow$ \\
\hline & \multicolumn{8}{|c|}{$\begin{array}{l}\text { without tc-MEP response (no amplitude presence) } \\
\text { baseline/endline }\end{array}$} \\
\hline & \multicolumn{8}{|c|}{ Number of patients ( \%) } \\
\hline TA 1.dx. & $0(0) / 2(7)$ & $1(7) / 0(0)$ & $1(100) / 1(100)$ & $0(0) / 1(33)$ & $5(38) / 6(46)$ & $8(67) / 8(67)$ & $10(40) / 11(44)$ & $15(20) / 19(25)$ \\
\hline AH 1.dx. & $0(0) / 1(3)$ & $1(7) / 0(0)$ & $1(100) / 1(100)$ & $0(0) / 1(33)$ & $4(31) / 5(38)$ & $8(67) / 8(67)$ & $9(36) / 10(40)$ & $14(19) / 18(24)$ \\
\hline Total base/end line & $0 \% / 6 \%$ & $8 \% / 8 \%$ & $100 \% / 100 \%$ & $0 \% / 25 \%$ & $35 \% / 42 \%$ & $71 \% / 71 \%$ & $40 \% / 46 \%$ & $15(20 \%) / 20(27 \%)$ \\
\hline Total mean & $3 \%$ & $8 \%$ & $100 \%$ & $13 \%$ & $38 \%$ & $71 \%$ & $43 \%$ & $24 \%$ \\
\hline \multicolumn{9}{|l|}{ Tc-MEP depend. on time } \\
\hline$\mu \mathrm{V} /$ anesthesia time & $27 \nearrow / 298$ & $1 \searrow / 323$ & $0 \searrow / 360$ & $65 \searrow / 340$ & $65 \searrow / 261$ & $207 \searrow / 228$ & $66 \searrow / 267$ & $62 \searrow / 288$ \\
\hline$\mu \mathrm{V} /$ minute & $0.09 \nearrow$ & $0.004 \searrow$ & $0 \searrow$ & $0.19 \searrow$ & $0.25 \searrow$ & $0.91 \searrow$ & $0.25 \searrow$ & $0.22 \searrow$ \\
\hline
\end{tabular}

Values are presented as a number (\%). The difference between the baseline mean value and the endline of tc-MEPs. $\nearrow$ amplitude increase, $\searrow$ amplitude decrease. Anesthesia time in minutes.

remifentanil, and sufentanil (magnitude increase $20 \%$ ) and bolus sufentanil (30\% decrease of magnitude), and bolus ketamine (730\% increase, $\mathrm{p}<0.008)$. Major differences were observed between bolus sufentanil (30\% decrease of magitude) and bolus ketamine $(730 \%$ increase of magnitude, $\mathrm{p}<0.001)$.

Anesthesia regimen in association without tc-MEP amplitude presence, outcome, percentage of patients

No significant difference was found between the groups of propofol (26\%), remifentanil (24\%), and sufentanil (28\%, p < $0.007)$. Considerable difference was found between the groups of propofol, remifentanil, and sufentanil (mean $26 \%$ ) and the group where ketamine boluses were administered. We didn't observe any loss of amplitude $(0 \%, \mathrm{p}<0.0002)$. In each comparison (propofol, remifentanil, and sufentanil) of the AIS2 and AIS3 groups (13\%) and between the AIS4, I, and ND groups, we found a higher incidence of no amplitude presence $(61 \%, \mathrm{p}<0.02)$.

Anesthesia regimen in association with spinal deformity along with tc-MEP amplitude changes, outcome

Significant similarity was found between the groups of propofol, remifentanil, and sufentanil and AIS3 and 4, N, D, I, and ND $(p<0.009)$. Difference was found in the groups of propofol, remifentanil, and sufentanil in AIS2 (41\% amplification of magnitude, $\mathrm{p}<0.04$ ) as compared to the groups of propofol, remifentanil, and sufentanil in AIS3 and 4, N, D, I, and ND (28\% decrease of magnitude). In all anesthesia groups (propofol, remifentanil, and sufentanil), significant decreases of tc-MEP amplitude were found in groups AIS4 and I $(74 \%, p<0.0001)$ compared to groups AIS2 and $3, \mathrm{~N}, \mathrm{D}, \mathrm{ND}$. 
Tab. 8. Anesthesia regimen in association with/without tc-MEPs between spinal deformities.

\begin{tabular}{|c|c|c|c|c|c|c|c|c|}
\hline \multirow{2}{*}{ Anesthetics } & AIS2 & AIS3 & AIS4 & $\mathrm{N}$ & $\mathrm{D}$ & I & ND & Total \\
\hline & \multicolumn{8}{|c|}{ mean tc-MEP magnitude baseline/endline } \\
\hline \multicolumn{9}{|l|}{ Sufentanil } \\
\hline TA 1.sin. & $148 \nearrow(64)$ & $11 \searrow(6)$ & $0(0)$ & $70 \nearrow(114)$ & $111 \nearrow(287)$ & $147 \searrow(52)$ & $22 \searrow(64)$ & $89 \lambda(43)$ \\
\hline AH 1.sin. & $151 \lambda(92)$ & $64 \searrow(25)$ & $0 \searrow(0)$ & 107\(62) & $613 \nearrow(3606)$ & $261 \searrow(76)$ & 29\(83) & $63 \lambda(33)$ \\
\hline TA l.dx. & $897(34)$ & $8 \searrow(5)$ & $0 \searrow(0)$ & $53 \nearrow(36)$ & $163 \nearrow(129)$ & $78 \searrow(70)$ & $10 \nearrow(35) \searrow$ & $51 \lambda(22)$ \\
\hline AH 1.dx. & $132 \nearrow(49)$ & $115 \searrow(38)$ & $0 \searrow(0)$ & $272 \searrow(81)$ & $75 \nearrow(80)$ & $195 \searrow(84)$ & $16 \searrow(8)$ & $19 \lambda(7)$ \\
\hline mean & $130 \pi(59)$ & $50 \searrow(19)$ & $0 \searrow(0)$ & $64 \searrow(1)$ & $240 \nearrow(1026)$ & $170 \searrow(71)$ & $14 \searrow(48)$ & $55 \lambda(26)$ \\
\hline & \multicolumn{8}{|c|}{$\begin{array}{l}\text { without tc-MEP response (no amplitude presence) } \\
\text { baseline/endline }\end{array}$} \\
\hline & \multicolumn{8}{|c|}{ Number of patients ( \%) } \\
\hline TA 1.sin. & $2(6) / 7(21)$ & $4(36) / 4(36)$ & $2(100) / 2(100)$ & $1(20) / 1(20)$ & $1(33) / 0(0)$ & $4(44) / 3(33)$ & $9(50) / 9(50)$ & $15(23) / 24(38)$ \\
\hline AH 1.sin. & $3(9) / 6(18)$ & $3(27) / 3(27)$ & $1(50) / 1(50)$ & $1(20) / 1(20)$ & $1(33) / 0(0)$ & $4(44) / 3(33)$ & $9(50) / 8(44)$ & $14(22) / 20(31)$ \\
\hline TA 1.dx. & $3(9) / 2(6)$ & $3(27) / 3(27)$ & $1(50) / 1(50)$ & $2(40) / 2(40)$ & $1(33) / 0(0)$ & $4(44) / 4(44)$ & $10(56) / 8(44)$ & $15(23) / 17(27)$ \\
\hline AH 1.dx. & $4(12) / 8(24)$ & $3(27) / 3(27)$ & $1(50) / 1(50)$ & $2(40) / 2(40)$ & $1(33) / 0(0)$ & $4(44) / 4(44)$ & $11(61) / 8(44)$ & $16(25) / 24(38)$ \\
\hline Total base/endline & $9 \% / 17 \%$ & $30 \% / 30 \%$ & $63 \% / 63 \%$ & $30 \% / 30 \%$ & $33 \% / 0 \%$ & $44 \% / 39 \%$ & $54 \% / 46 \%$ & $15(23 \%) / 21(33 \%)$ \\
\hline Total mean & $13 \%$ & $30 \%$ & $63 \%$ & $30 \%$ & $17 \%$ & $42 \%$ & $50 \%$ & $28 \%$ \\
\hline \multicolumn{9}{|l|}{ Tc-MEP depend. on time } \\
\hline$\mu \mathrm{V} /$ anesthesia time & $75 \pi / 277$ & $64 \searrow / 310$ & $218 \searrow / 375$ & $75 \searrow / 368$ & $95 \pi / 278$ & $104 \searrow / 250$ & $18 \searrow / 296$ & $18 \pi / 289$ \\
\hline$\mu \mathrm{V} /$ minute & $0.270 \nearrow$ & $0.207 \searrow$ & $0.581 \searrow$ & $0.204 \backslash$ & $0.343 \pi$ & $0.415 \searrow$ & $0.062 \searrow$ & $0.063 \pi$ \\
\hline
\end{tabular}

Values are presented as a number (\%). The difference between the baseline mean value and endline of tc-MEPs. $\nearrow$ amplitude increase, $\searrow$ amplitude decrease. Anesthesia time in minutes.

Tab. 9. Anesthesia regimen in association with/without tc-MEPs between spinal deformities.

\begin{tabular}{|c|c|c|c|c|c|c|c|c|}
\hline \multirow{2}{*}{ Anesthetics } & AIS2 & AIS3 & AIS4 & $\mathrm{N}$ & $\mathrm{D}$ & I & ND & Total \\
\hline & \multicolumn{8}{|c|}{ mean tc-MEP baseline $/ 30 \mathrm{~min}$} \\
\hline \multicolumn{9}{|l|}{ Bolus sufentanil } \\
\hline TA 1.sin. & $107 \searrow(54)$ & $68 \searrow(70)$ & $0(0)$ & $579 \searrow(52)$ & $21 \searrow(47) \nearrow$ & $111 \searrow(52)$ & 144\(49) & 109\(49) \\
\hline AH 1.sin. & $52 \searrow(50)$ & 79\(45) & $0 \searrow(0)$ & $65 \searrow(10)$ & $21 \searrow(61)$ & $243 \searrow(77)$ & $81 \searrow(58)$ & $71 \searrow(50)$ \\
\hline TA l.dx. & $71 \searrow(30) \nearrow$ & $74 \searrow(55)$ & $0 \searrow(0)$ & $255 \searrow(34)$ & $90 \nearrow(36)$ & $210 \searrow(87)$ & $76 \searrow(36)$ & $77 \searrow(1)$ \\
\hline AH l.dx. & 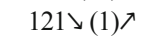 & $240 \searrow(68)$ & $0 \searrow(0)$ & $1746 \searrow(75)$ & $24 \lambda(18)$ & $328 \searrow(89)$ & $280 \searrow(45)$ & $216 \searrow(24)$ \\
\hline \multirow[t]{3}{*}{ mean } & $88 \searrow(17)$ & $115 \searrow(60)$ & $0 \searrow(0)$ & $661 \searrow(43)$ & $18 \lambda(10)$ & $223 \searrow(76)$ & $145 \searrow(47)$ & $118 \searrow(30)$ \\
\hline & \multicolumn{8}{|c|}{$\begin{array}{l}\text { without tc-MEP response (no amplitude presence) } \\
\text { baseline/30min }\end{array}$} \\
\hline & \multicolumn{8}{|c|}{ Number of patients ( $\%)$} \\
\hline TA 1.sin. & $2(13) / 2(13)$ & $0(0) / 3(50)$ & $0(0) / 0(0)$ & $0(0) / 0(0)$ & $0(0) / 1(50)$ & $0(0) / 0(0)$ & $1(10) / 4(40)$ & $2(8) / 6(23)$ \\
\hline AH 1.sin. & $2(13) / 3(20)$ & $0(0) / 1(17)$ & $0(0) / 0(0)$ & $0(0) / 0(0)$ & $0(0) / 1(50)$ & $0(0) / 0(0)$ & $1(10) / 3(30)$ & $2(8) / 5(19)$ \\
\hline TA l.dx. & $1(7) / 2(13)$ & $0(0) / 1(17)$ & $0(0) / 0(0)$ & $0(0) / 0(0)$ & $0(0) / 0(0)$ & $0(0) / 0(0)$ & $1(10) / 1(10)$ & $1(4) / 3(12)$ \\
\hline AH l.dx. & $2(13) / 6(40)$ & $0(0) / 1(17)$ & $0(0) / 0(0)$ & $0(0) / 0(0)$ & $0(0) / 0(0)$ & $0(0) / 0(0)$ & $1(10) / 1(10)$ & $2(8) / 7(27)$ \\
\hline Total base/30min & $12 \% / 12 \%$ & $0 \% / 13 \%$ & $0 \% / 0 \%$ & $0 \% / 0 \%$ & $0 \% / 13 \%$ & $0 \% / 0 \%$ & $10 \% / 18 \%$ & $2(7 \%) / 5(11 \%)$ \\
\hline Total mean & $12 \%$ & $6 \%$ & $0 \%$ & $0 \%$ & $6 \%$ & $0 \%$ & $14 \%$ & $9 \%$ \\
\hline \multicolumn{9}{|l|}{ Tc-MEP dependent on time } \\
\hline$\mu \mathrm{V} / 30 \mathrm{~min}$ anesthesia time & $88 \searrow / 30$ & $115 \searrow / 30$ & $0 \searrow / 0$ & $661 \searrow / 30$ & $18 \pi / 30$ & $223 \searrow / 30$ & $145 \searrow / 30$ & $214 \searrow / 30$ \\
\hline$\mu \mathrm{V} /$ minute & $2.92 \searrow$ & $3.83 \searrow$ & $0 \searrow$ & $22.04 \backslash$ & $0.60 \pi$ & 7.43】 & $4.84 \searrow$ & 7.13】 \\
\hline
\end{tabular}

Values are presented as a number (\%). The difference between the baseline mean value and after 30 minutes of tc-MEPs. $\nearrow$ amplitude increase, $\searrow$ amplitude decrease. Anesthesia time in minutes.

Anesthesia regimen in association with anesthesia time along with tc-MEP amplitude change, outcome

The average amplitude of tc-MEPs decreased in the groups of propofol, remifentanil, and sufentanil (35\% decrease of magnitude) during the average 288 minutes of anesthesia time. We didn't consider 2 patients in sufentanil group D because of tcMEP excitability.

Deformity types in association with tc-MEP changes, outcome

In this group, there were 139 patients $(100 \%)$ divided into spinal deformity subgroups (Table 2). Significant differences ap- peared in age between groups AIS2-4, and N (18 years old) and groups D, I, and ND (51 years old, $\mathrm{p}<0.0005)$. Sex differences were observed in scoliotic groups AIS2-4, N, and D (88 \% F) and group I without scoliotic deformities $(52 \% \mathrm{~F}, \mathrm{p}<0.001)$. Tc-MEP differences were found between groups AIS2 (magnitude increase $41 \%$ ) and AIS3, AIS4, N, D, and I (mean magnitude decrease $34 \%, \mathrm{p}<0.02$ ). Major differences were found between groups AIS2 and 3, N, and D (mean magnitude increase $5 \%$ ) and groups AIS4 and I (mean magnitude decrease $74 \%, \mathrm{p}$ $<0.004$ ) (Tab. 6). 
Tab. 10. Anesthesia regimen in association with/without tc-MEPs between spinal deformities.

\begin{tabular}{|c|c|c|c|c|c|c|c|c|}
\hline \multirow{2}{*}{ Anesthetics } & AIS2 & AIS3 & AIS4 & $\mathrm{N}$ & $\mathrm{D}$ & I & ND & Total \\
\hline & \multicolumn{8}{|c|}{ mean tc-MEP baseline/30min } \\
\hline \multicolumn{9}{|l|}{ Bolus ketamine } \\
\hline TA1.sin. & $197 \nearrow(310 \%)$ & $52 \lambda(67)$ & $0(0)$ & $732 \lambda(247)$ & $0(0)$ & 99\(19) & $257 \nearrow(200)$ & $195 \nearrow(278)$ \\
\hline $\mathrm{AH}$ 1.sin. & $146 \nearrow(188)$ & $2 \searrow(4)$ & $0(0)$ & $440 \searrow(72)$ & $0(0)$ & $75 \searrow(18)$ & $13 \nearrow(152)$ & $107 \nearrow(157)$ \\
\hline TA 1.dx. & $235 \nearrow(1289)$ & $38 \lambda(36)$ & $0(0)$ & $2256 \lambda(738)$ & $0(0)$ & $10 \nearrow(11)$ & $442 \lambda(154)$ & $285 \nearrow(1136)$ \\
\hline AH 1.dx. & $243 \pi(1558)$ & $14 \lambda(31)$ & $0(0)$ & $522 \searrow(56)$ & $0(0)$ & $14 \nearrow(350)$ & $47 \searrow(68)$ & $191 \nearrow(1348)$ \\
\hline mean & $205 \lambda(836)$ & $25 \lambda(33)$ & $0(0)$ & $507 \lambda(226)$ & $0(0)$ & $38 \searrow(81)$ & $166>(143)$ & $195 \lambda(730)$ \\
\hline & \multicolumn{8}{|c|}{$\begin{array}{c}\text { without tc-MEP response (no amplitude presence) } \\
\text { baseline/30min }\end{array}$} \\
\hline & \multicolumn{8}{|c|}{ Number of patients ( \%) } \\
\hline Total base/30min & $0 \%$ & $0 \%$ & $0 \%$ & $0 \%$ & $0 \%$ & $0 \%$ & $0 \%$ & $0 \%$ \\
\hline \multicolumn{9}{|l|}{ Tc-MEP dependent on time } \\
\hline$\mu \mathrm{V} / 30 \mathrm{~min}$ anesthesia time & $205 \lambda / 30$ & $25 \pi / 30$ & $0 / 0$ & $507 \nearrow / 30$ & $0 / 0$ & $38 \searrow / 30$ & $166 \pi / 30$ & $195 \pi / 30$ \\
\hline$\mu \mathrm{V} /$ minute & $6.84 \nearrow$ & $0.84 \lambda$ & 0 & $16.88 \pi$ & 0 & $1.25 \searrow$ & $5.54 \nearrow$ & $6.48 \pi$ \\
\hline
\end{tabular}

Values are presented as a number (\%). The difference between the baseline mean value and after 30 minutes of tc-MEPs. $\nearrow$ amplitude increase, $\searrow$ amplitude decrease. Anesthesia time in minutes. Bolus ketamine table doesn't have patients without a tc-MEP response.

Neurological deficit, anesthesia, outcome, percentage of patients

The success rate of tc-MEP recording according to the neurological deficit group in the propofol, remifentanil, and sufentanil groups was $17 \%$ in MRC 3, $96 \%$ in MRC 4, and $87 \%$ in MRC 5. In the neurological deficit (ND) group without tc-MEP amplitude, the response mean was $46 \%$ patients in the propofol, remifentanil and sufentanil groups.

\section{Discussion}

Intraoperative neurophysiological monitoring using tc-MEPs is routinely employed in spinal surgery, particularly for procedures involving scoliosis correction, spinal neoplasms and other spinal deformities. There are known limitations to IONM. Multiple non-neurological related factors can affect the waveforms, such as blood pressure, heart rate, temperature, partial pressure of alveolar carbon dioxide, and anesthetic drugs (4). In addition, challenging clinical conditions, such as severe myelopathy, obesity, or peripheral neuropathy can make interpretation difficult or even impossible (35). In our study we observed that in the group with neurological deficit (ND) no tc-MEPs appeared in 20 patients (14 $\%$ ) out of a total of 139 patients (100\%). This $14 \%$ of patients divided into subgroups where 13 patients $(9 \%)$ had a new neurological deficit and the other 7 patients (5\%) didn't experience any new change in neurological deficit. Other studies have also found similar results. Clark et al (36) found that neurophysiological alerts were present in 16 patients $(11 \%)$ out of a total of 140 patients. Overall, 8 patients $(6 \%)$ experienced new postoperative neurological deficits. There was a significant association between IONM MEP alerts and the presence of a new postoperative deficit.

In studies of patients undergoing scoliosis surgery, the incidence of a significant intraoperative monitoring change has been reported to occur from $3.6 \%$ to $8.7 \%(37-39)$. Another study recorded an increased incidence of $11.8 \%$ of patients with a neurological deficit before surgery worsening postoperatively (25).

A total of 16 patients (17\%) recorded a successful response of tc-MEPs in our group with neurological deficit according to MRC grade 3. In another study, Lee et al (40) successfully obtained basal tc-MEP amplitude according to MRC grade 3 in 4 patients (28.6 $\%)$. Any muscle amplitude MEPs in the lower extremities was considered a successful recording.

Reports suggest that tc-MEP responses degrade or undergo anesthetic fade during surgery despite unchanged anesthetic levels and other physiologic variables (5). In a study by Ugawa et al (41), the amplitude of MEP significantly decreased in the lower limbs at 5 hours by $31 \%$ and at 6 hours by $39 \%$ after the initial infusion of propofol. Mean operating time was 282.1 minutes. We considered significant similarities in our study. Mean tc-MEP amplitude decreased in the groups of propofol, remifentanil and sufentanil by $35 \%$ in an average of 288 minutes of anesthesia time $(\mathrm{p}<0.04)$. Lonner et al $(42)$ reported that the operating time for the treatment of adolescent idiopathic scoliosis was $180.2 \mathrm{~min}$ for experienced surgeons and 221.9 min for inexperienced surgeons. In comparison to our study group of adolescent idiopathic scoliosis AIS2, there was $287 \mathrm{~min}$ of anesthesia time. If we consider the real operating time it would be $204 \mathrm{~min}$. In our study, the time between the induction of anesthesia and the surgery is on average $63 \mathrm{~min}$. The time of surgery end and anesthesia end is on average $20 \mathrm{~min}$.

Tc-MEPs tend to exhibit gradually rising thresholds during the hours of surgery. Sedative-hypnotics such as propofol cause unconsciousness by producing corticocortical inhibition, possibly by GABA-mediated (Gamma-aminobutyric acid) inhibitory interneuron activity within the cerebral cortex with minimal depression of spinal alpha motor neurons. Propofol produces a dose-dependent depression of tc-MEP responses.

The mechanism of action of ketamine (ket) is rather complex. Moderate doses of ket are thought to increase motor excitability by blocking $N$-methyl-D-aspartate (NMDA) receptor-mediated excitatory inputs to inhibitory interneurons $(18,43,44)$. Furmaga et al (19) found in their study that cortical excitability, as indexed by the amplitude of MEPs, was higher under ket than propofol. Our study showed retrospectively that administration of a $30 \mathrm{mg}$ ketamine bolus during surgery increased the tc-MEP amplitude after mean 30 minutes to $195 \mu \mathrm{V}(730 \%)$. 
794-801

In older groups, the success rate can decrease due to peripheral neuropathy or age-related disorders (40). In our study, we confirmed this statement. The group of patients in AIS2, with a mean age of 17 , had 2 patients ( $3 \%$ ) with neurological deficit and no tc-MEP amplitude in 5 patients $(8 \%)$. Overall, a significant increase of tc-MEP amplitude $83 \mu \mathrm{V}(41 \%)$ was observed successfully in 59 patients $(92 \%, \mathrm{p}<0.0005)$. Older patients with a mean age of 51 in groups D, I, and ND underwent anesthetic fade of $38 \%$ amplitude decrease.

Many cases in our study with significant tc-MEP alerts underwent an evidence-based response checklist (45).

\section{Conclusion}

We found that intraoperative neurophysiological monitoring can be performed with either way of TIVA: propofol, remifentanil and sufentanil, with no statistical difference. Significant findings were found in the administration of sufentanil boluses where the amplitude decreased and in ketamine boluses where the amplitude rose. We also confirmed the claims of anesthetic fade in tc-MEP amplitude during long surgery. Spinal deformities with more severe curves had a magnitude decrease. In older patients and neurologic deficit patients we also found the same situation.

The effect of anesthetics on tc-MEPs is dose and time dependent. The usefulness of IONM is dependent on patient samples and accurate evaluation of tc-MEPs. The roles of the spinal surgeon, anesthesiologist and neurophysiologist are to cooperate in early recognition of tc-MEP change and find an accurate method to ensure the best neurological outcome.

\section{References}

1. Epstein NE, Danto J, Nardi D. Evaluation of intraoperative somatosensory-evoked potential monitoring during 100 cervical operations. Spine (Phila Pa 1976) 1993; 18: 737-747.

2. Gokaslan ZL, Samudrala S, Deletis V, Wildrick DM, Cooper PR. Intraoperative monitoring of spinal cord function using motor evoked potentials via transcutaneous epidural electrode during anterior cervical spinal surgery. J Spinal Disord 1997; 10: 299-303.

3. Papastefanou SL, Henderson LM, Smith NJ, Hamilton A, Webb JK. Surface electrode somatosensory-evoked potentials in spinal surgery: implication for indications and practise. Spine (Phila Pa 1976) 2000; 25 : $2467-2472$

4. Traynelis VC, Abode-Iyamah KO, Leick KM, Bender SM, Greenlee JD. Cervical decompression and reconstruction without intraoperative neurophysiological monitoring. Clinical article. J Neurosurg Spine 2012; 16: 107-113.

5. Lyon R, Feiner J, Lieberman JA. Progressive suppression of motor evoked potentials during general anesthesia: the phenomenon of ,anesthetic fade“. J Neurosurg Anesthesiol 2005; 17: 13-19.

6. Pelosi L, Lamb J, Grevitt M, Mehdian SM, Webb JK, Blumhardt LD. Combined monitoring of motor and somatosensory evoked potentials in orthopaedic spinal surgery. rev. Clin Neurophysiol 2002; 113: 1082-1091.
7. Lall RR, LallRR, Hauptman JS, Munoz C, Cybulski GR, Koski T et al. Intraoperative neurophysiological monitoring in spine surgery: indications, efficacy, and role of the preoperative checklist. Neurosurg Focs 2012; 33: E10.

8. Hilibrand AS, Schwarty DM, Sethuraman V, Vaccaro AR, Albert TJ. Comparison of transcranial electric motor and somatosensory evoked potential monitoring during cervicalspine surgery. J Bone Joint Surg Am 2004; 86-A: $1248-1253$.

9. Leslie K,Sessler DI, Bjorkset AR, Moayeri A. Mild hypothermia alters propofol pharmacokinetics and increases the duration of action of atracurium. Anesth Analg. 1995; 80: 1007-1014.

10. Othman Z, Lenke LG, Bolon SM, Padberg A. Hypotension-induced loss of intraoperative monitoring data during surgical correction of scheuermann kyphosis: a case report. Spine (Phila Pa 1976) 2004; 29 : E258-265.

11. Calancie B, Klose KJ, Baier S et al. Isoflurane-induced attenuation of motor evoked potentials caused by electrical motor cortex stimulation during surgery. J. Neurosurg. 1991; 74: 897-904.

12. Sekimoto K, Nishikawa K, Ishizeko J, Kubo K, Saito S, Goto F. The effects of volatile anesthetics on intraoperative monitoring of myogenic motor-evoked potentials to transcranial electrical stimulation and on partial neuromuscular blockade during propofol/fentanyl/nitrous oxide anesthesia in humans. J Neurosurg Anesthesiol 2006; 18: 106-111.

13. Liu EH, Wong HK, Chia CP, Lim HJ, Chen ZY, Lee TL. Effects of isoflurane and propofol on cortical somatosensory evoked potentials during comparable depth of anesthesia as guided by bispectral index. $\mathrm{Br}$ J Anaesth. 2005; 94(2): 193-197.

14. Chen Z. The effects of isoflurane and propofol on intraoperative neurophysiological monitoring during spinal surgery. J Clin Monit Comput 2004; 18: 303-308.

15. Di Lazzaro V, Oliviero A, Profice P, Pennisi MA, Pilato F, Zito G et al. Ketamine increases human motor cortex excitability to transcranial magnetic stimulation. J Physiol 2003; 547: 485-496.

16. Kalkman CJ, Drummond JC, Patel PM, Sano T, Chesnut RM. Effects of droperidol, pentobarbital and ketamine on myogenic transcranial magnetic motor-evoked responses in humans. Neurosurg 1994; 35 : 1066-1071.

17. Kothbauer K, Schmid UD, Liechti S, Rosler KM. The effect of ketamine anesthetic induction on muscle responses to transcranial magnetic cortex stimulation studied in man. Neurosci Lett 1993; 154: 105-108.

18. Ziemann U. TMS and drugs. Clin Neurophysiol 2004; 115: 1717-1729.

19. Furmaga H,Park HJ, Cooperrider J, Baker KB, Johnson M, Gale JT et al. Effects of ketamine and propofol on motor evoked potentials elicited by intracranial microstimulation during deep brain stimulation. Front in Syst Neurosci 2014; 89 (8): 1-9.

20. Kawaguchi M, Furuya H. Intraoperative spinal cord monitoring of motor function with myogenic motor evoked potentials: a consideration in anesthesia. J Anesth 2004; 18: 18-28.

21. Lo YL, Tan YE, Raman S, Teo A, Dan YF, Guo ChM. Systematic re-evaluation of intraoperative motor-evoked potential suppression in scoliosis surgery. Scoliosis and Spin Disorders 2018; 13 (12): 1-6.

22. Hayashi H, Kawaguchi M,Yamamoto Y,Inoue S, Koizumi M, Ueda Y. Evaluation of reliabilty of post-tetanic motor-evoked potential monitoring during spinal surgery under general anesthesia. Spine (Phila Pa 1976) 2008; 33E: 994-1000. 
23. Scheufler KM, Reinacher PC, Blumrich W, Zentner J, Priebe HJ. The modifying effects of stimulation pattern and propofol plasma concentration. Anesth Analg 2005; 100: 440-447.

24. MacDonald DB, Al Zayed Z, Khoudeir I, Stigsby B. Monitoring scoliosis surgery with combined multiple pulse transcranial electric motor and cortical somatosensory-evoked potentials from the lower and upper extremities. Spine (Phila Pa 1976) 2003; 28: 194-203.

25. Royan NP, Lu N, Manninen P, Venkatraghavan L. The influence of anaesthesia on intraoperative neuromonitoring changes in high-risk spinal surgery. J Neuroanaesth and Crit Care 2017; 3: 159-166.

26. Holdefer RN, Anderson C, Furman M, Sangare Y, Slimp JC. A comparison of the effects of desflurane versus propofol on transcranial motor-evoked potentials in pediatric patients. Childs Nerv Syst 2012; 30: $2103-2108$.

27. Liu HY, Zeng HY, CHeng H, Wang MR, Qiao H, Han RQ. Comparison of the effects of etomidate and propofol combined with remifentanil and guided by comparable BIS on transcranial electrical motor-evoked potentials during spinal surgery. J neurosurg Anesthesiol 2012; 24: 133-138.

28. Park JH, Hyun SJ. Intraoperative neurophysiological monitoring in spinal surgery. World J Clin Cases 2015; 3 (9): 765-773.

29. Sala F, Bricolo A, Faccioli F, Lanteri P, Gerosa M. Surgery for intramedullary spinal cord tumors: the role of intraoperative (neurophysiologica) monitoring. Eur Spine J 2007; 2: 130-139.

30. Macdonald DB, Skinner S, Shils J, Yingling C. Intraoperative motor evoked potential monitoring - a position statement by the American Society of Neurophysiological Monitoring. Clin Neurophysiol 2013; 124 : 2291-2316.

31. Kothbauer KF. Intraoperative neurophysiologic monitoring for intramedullary spinal-cord tumor surgery. Neurophysiol Clin 2007; 37: 407-414.

32. Hyun SJ, Rhim SC. Combined motor and somatosensory evoked potntial monitoring for intramedullary spinal cord tumor surgery: correlation of clinical and neurophysiological data in 17 consecutive procedures. Br J Neurosurg 2009; 23: 393-400.

33. Hyun SJ, Rhim SC, Kang JK, Hong SH, Park BR. Combined motor and somatosensory-evoked potntial monitoring for spine and spinal cord surgery: correlation of clinical and neurophysiological data in 85 consecutive procedures. Spinal Cord 2009; 47: 616-622.
34. https://math.feld.cvut.cz/novotny/1617/PMS/tabulky.pdf.

35. Schwartz DM, Sestokas AK, Turner LA, Morledge DE, DiNardo AA Jr, Beacham SG. Neurophysiological identification of iatrogenic neural injury during complex spine surgery. Semin Spine Surg 1998; 10: $242-251$.

36. Clark AJ, Ziewacz JE, Safaee $M$ et al. Intraoperative neuromonitoring with MEPs and prediction of postoperative neurological deficits in patients undergoing surgery for cervical and cervicothoracic myelopathy. Neurosurg Focus 2013; 35 (1): 1-8.

37. Buckwalter JA, Yaszay B, Ilgenfritz RM, Bastrom TP, Newton PO. Analysis of intraoperative neuromonitoring events during spinal corrective surgery for idiopathic scoliosis. Spine Deform 2013; 1: 434-8.

38. Samdani AF, Bennett JT, Ames RJ, Asghar JK, Orlando G, Pahys JM et al. Reversible intraoperative neurophysiologic monitoring alerts in patients undergoing arthrodesis for adolescent idiopathic scoliosis: What are the outcomes of surgery? J Bone Joint Surg Am 2016; 98: 1478-83.

39. Polly DW Jr., Rice K, Tamkus A. What is the frequency of intraoperative alerts during pediatric spinal deformity surgery using current neuromonitoring methodology? A retrospective study of 218 surgical procedures. Neurodiagn J 2016; 56: 17-31.

40. Lee JM, Kim DH, Kim HS, Choi BK, Han IH. The Applicability of Intraoperative Neuromonitoring in Patients with Preoperative Motor Weakness during Spine Surgery. Korean J Spine 2016; 13 (1): 9-12.

41. Ugawa R, Takigawa T, Shimomiya $H$ et al. An evaluation of anesthetic fade in motor evoked potential monitoring in spinal deformity surgeries. J Orthop Surg Res 2018; 13 (227): 1-6.

42. Lonner BS, Auerbach JD, Estreicher MB, Kean KE. Thoracic pedicle screw instrumentation: the learning curve and evolution in technique in the treatment of adolescent idiopatic scoliosis. Spine 2009; 34: 2158-64.

43. Brown EN, Lydic R, Schiff ND. General anesthesia, sleep and coma. N Engl J Med 2010; 363: 2638-50.

44. Brown EN, Purdon PL, Van Dort CJ. General anesthesia and altered states of arousal: a systems neuroscience analysis. Annu Rev Neurosci 2011; 34: 601-28.

45. Ziewacz JE, Berven SH, Mummaneni VP, Tu TH, Akinbo OC, Lyon $\mathbf{R}$ et al. The design, development, and implementation of a checklist for intraoperative neuromonitoring changes. Neurosurg Focus 2012; 33 (5): E11.

Received June 4, 2019.

Accepted July 17, 2019. 